\title{
Limit Spectra of Random Gram Matrices
}

\author{
V.I. SERDOBOLSKII
}

Moscow State Institute of Electronics and Mathematics, Moscow 109028, B. Trekhsviatitelskii 3, Russia

E-mail:vserd@mail.ru

This article is part of the Proceedings titled "Geometrical Mathods in Physics: Bialowieza XXI and XXII"

\begin{abstract}
Solutions to basic non-linear limit spectral equation for matrices $R^{T} R$ of increasing dimension are investigated, where $R$ are rectangular random matrices with independent normal entries. The analytical properties of limiting normed trace for the resolvent of $R^{T} R$ are investigated, boundaries of limit spectra found, and upper estimates of spectral density are obtained.
\end{abstract}

Spectra of large random Gram matrices are of interest in "random matrix physics" (see [1]), and in solution of systems of empiric linear algebraic equations.

First, we cite shortly some recent results stating the convergence of spectral functions obtained earlier. The basic spectral equation (called "canonic equation" by V.I. Girko [2]) is established that connect limit spectral functions of random Gram matrices with limit spectral function of non-random matrices. Then we suggest an original investigation of solutions of this spectral equation and of the form of limit spectra with full proofs.

We consider a sequence $\mathbf{P}$ of matrices $A$ and $R$ of size $N \times n, \xi=R-A$, and square matrices $\Sigma=A^{T} A, \quad S=R^{T} R$ of size $n \times n$ as $n \rightarrow \infty, N \rightarrow \infty$, where $\xi$ are random vectors with independent components Denote by $A_{m j}, R_{m j}, \xi_{m j}$ entries of $A, R, \xi, \quad m=1, \ldots, N, \quad j=1, \ldots, n$.

Assume the following.

1. For each $n$ the inequality holds $n \leq N$.

2. For each $n$ the spectral norm $\|A\|^{2} \leq c_{1}$, where $c_{1}$ does not depend on $n$.

3. As $n \rightarrow \infty$ the magnitudes $\kappa_{n}=N / n-1 \rightarrow \kappa \geq 0$.

4. Entries $\xi_{m i}$ of the matrix $\xi$ are independent and normally distributed as $\mathbf{N}(\mathbf{0}, \mathbf{d} / \mathbf{n})$, $m=1 \ldots, N, \quad i=1, \ldots, n$. Let $d$ be independent of $n$.

We apply methods developed in the spectral theory of random matrices of increasing dimension $[2,3]$. Let us study the resolvent

$$
H=H(t)=(S+t I)^{-1}, \quad t \geq 0,
$$

and spectral functions depending on $H(t)$.

To deduce the basic spectral equation, first, we need upper estimates of variance of some spectral functions. 
Lemma 1. Under assumptions 1-4 for $t \geq c>0$ the variance

$\operatorname{var}\left(n^{-1} \operatorname{tr} H(t)\right) \leq k N / n^{3}, \quad$ where $\quad k=a d\left(c_{1}+d\right)\left[c^{2}+\left(c_{1}+d\right)^{2}\right] / c^{8}$,

where $k$ is a numeric coefficient.

Lemma 2. Under assumptions 1-4 for any non-random unit vectors $\mathbf{e}$ and $t \geq c>0$ as $n \rightarrow \infty$ uniformly

$\operatorname{var}\left(\mathbf{e}^{T} H(t) \mathbf{e}\right)=O\left(n^{-1}\right)$.

Denote $h_{n}=n^{-1} \operatorname{tr} H, s_{n}=1+h_{n} d, r_{n}=t s_{n}+\kappa_{n} d$.

We also need following asymptotic relations.

Lemma 3. Under assumptions $1-4$ for $t \geq c>0$ as $n \rightarrow \infty$ uniformly

$\mathbf{E} H R^{T}=\mathbf{E} H A^{T} / s_{n}+\Omega$,

$\mathbf{E} H R^{T} R=\mathbf{E} H R^{T} A+d\left(\kappa_{n}+h_{n} t\right) \mathbf{E} H+O\left(n^{-1}\right)$.

where $\|\Omega\|=O\left(n^{-1}\right)$.

Suppose additionally that the weak convergence holds

$$
F_{0 n}(u) \stackrel{\text { def }}{=} n^{-1} \sum_{i=1}^{n} \text { ind }\left(\lambda_{0 i} \leq u\right) \rightarrow F_{0}(u), \quad u \geq 0,
$$

where $\lambda_{0 i}$ are eigenvalues of matrices $A^{T} A, i=1, \ldots, n$.

From Lemmas 1-3 the following theorem can be stated establishing asymptotic relations between spectra of random matrices $R^{T} R$ and spectra of non-random matrices $A^{T} A$ (V.I.Serdobolskii and A.V.Serdobolskii, 1991).

Theorem 1. Under assumptions 1-4 and (1) the following is true.

1. For $t \geq c>0$ the convergence in the square mean holds

$$
h_{n}=h_{n}(t) \stackrel{\text { def }}{=} n^{-1} \operatorname{tr} H(t) \rightarrow h(t), \quad r_{n}=r_{n}(t) \stackrel{\text { def }}{=} t s_{n}(t)+\kappa_{n} d \rightarrow r(t),
$$

uniformly with respect to $t \geq c>0$, where $s(t)=1+d h(t)$, and $r(t)=t s(t)+\kappa d$.

2. For each $t \geq 0$ the equation holds

$$
h(t)=s(t) \int(u+r(t) s(t))^{-1} d F_{0}(t) .
$$

3. For $n \rightarrow \infty$ the weak convergence in the square mean holds

$$
F_{n}(u) \stackrel{\text { def }}{=} n^{-1} \sum_{i=1}^{n} \text { ind }\left(\lambda_{i} \leq u\right) \stackrel{P}{\rightarrow} F(u),
$$

where $\lambda_{i}$ are eigenvalues of $R^{T} R, i=1, \ldots, n$. 
4. For each $t>0$ the equation holds

$$
h(t)=\int(u+t)^{-1} d F(u) .
$$

5. As $n \rightarrow \infty$

$$
\mathbf{E} H(t)=s(t)\left(A^{T} A+r(t) s(t)\right)^{-1}+\Omega_{n},
$$

where $\left\|\Omega_{n}\right\|=O\left(n^{-1}\right)$ uniformly with respect to $t \geq c>0$.

The limit equation (2) can be called basic spectral equation in the limit spectral theory of random Gram matrices. In this paper we use this equation for studying properties of limit spectra.

\section{Limit spectra}

Theorem 2. Under assumptions 1-4 and (1) the following is true.

1. The function $h(t)$, defined by (1) for $t \geq c>0$, allows the analytical continuation for complex arguments $z$; this function is regular everywhere except the half-axis $z \leq 0$, is uniformly bounded and has a uniformly bounded derivative inside of any compact not containing points of $z \leq 0$. If $\kappa>0$, then $h(z)$ is regular also around $z=0$ and $c_{2}^{-1} \leq h(0) \leq 1 /(\kappa d)$.

2. For any $v=-\operatorname{Re} z>0$ and $\varepsilon=-\operatorname{Im} z \rightarrow+0$ there exists the limit $\lim \pi^{-1} \operatorname{Im} h(z)=$ $F^{\prime}(v)$.

3. For $v>0 \quad F^{\prime}(v) \leq \pi^{-1}(v d)^{-1 / 2}$.

Consider the set $\mathbf{S}=\left\{v>0: F^{\prime}(v)>0\right\}$ ("matrices $S$ limit spectral region").

4. If $\kappa>0$, then the set $\mathbf{S}$ of real $z=-v$ is located on the segment, on which $v \in\left[v_{1}, v_{2}\right]$, where

$$
v_{1}=\frac{\kappa^{2} d}{4(\sqrt{1+\kappa}+1)^{2}}, \quad v_{2}=\max \left[9 d,\left(1+\frac{\kappa}{2}\right) d+\frac{9}{8} c_{2}\right]
$$

the diameter of $\mathbf{S}$ is not less than $\pi d \kappa /[4(1+\sqrt{1+\kappa})]$.

5. If $\kappa>0$, then for all $v \geq 0$ the function $F^{\prime}(v)$ satisfies the Lipschitz condition with the exponent $1 / 3$ and is differentiable everywhere, perhaps, except the boundary points of the set $\mathbf{S}$.

6. If $\kappa>0$, then the function $h(z)$ satisfies the Goelder condition on the whole plane of complex $z$ with the exponent $1 / 3$.

Proof. Starting from (3) one can easily establish the possibility of analytical continuation of $h(z)$, its uniform boundedness, regularity and uniform boundedness of the derivative outside any vicinity of $\mathbf{S}$. From (3) one also can see that the derivative $s^{\prime}(z)$ exists and is uniformly bounded there. If $\kappa>0$ then $v_{1}>0$ and the right boundary of $\mathbf{S}$ lays at 
a finite distance from $z=0$. Lower and upper boundaries for $h(0)$ can be easily derived from (3) and (2). The first statement of our theorem is proved.

To be concise, denote $h=h(z), s=s(z)=1+h d, r=r(z)=z s+\kappa d, \quad z_{0}=\operatorname{Re} z$, $z_{1}=\operatorname{Im} s$, and let

$$
\mu_{\nu}=\int|v+r s|^{-2} v^{\nu} d F_{0}(v), \quad \nu=1,2 .
$$

Let us fix some $v=-\operatorname{Re} z>0$ and tend $z=-v-i \varepsilon$ to $-v, \varepsilon \rightarrow+0$.

If $v \in \mathbf{S}$ ("on the spectrum"), $\operatorname{Im} h(z) \rightarrow \pi F^{\prime}(v)>0$. We divide both parts of (2) by $s$, take imaginary parts, and obtain

$$
1 / \mu_{0}=p|s|^{2} d+O(\varepsilon), \quad v \in \mathbf{S},
$$

where $|s| \geq d \operatorname{Im} h \rightarrow d \pi F^{\prime}(v)>0$. Taking imaginary parts both in the left-hand and in the right-hand sides we find that $1 / \mu_{0}=d \mu_{1} / \mu_{0}+d|s|^{2} v+O(\varepsilon)$. From these two equations it follows that

$$
|s|^{2}(p-v)=\mu_{1} / \mu_{0}+O(\varepsilon), \quad v \in \mathbf{S} .
$$

Since $|s| \geq\left|s_{1}\right| \rightarrow \pi d F^{\prime}(v)>0$ and $v>0$ we conclude that $p>v+O(\varepsilon)$.

Further, using the Cauchy-Buniakovsky inequality from (2) we find that $|h|^{2} \leq \mu_{0}|s|^{2}$. Substituting $\mu_{0}$ from (4) we obtain $|h|^{2} p d \leq 1+O(\varepsilon)$ and

$$
|s-1|^{2} \leq d / p+O(\varepsilon) \leq d / v+O(\varepsilon) .
$$

In particular, it implies that the variable $\operatorname{Im} h \leq 1 / \sqrt{v d}+O(\varepsilon)$ for $v \in \mathbf{S}$ and $F^{\prime}(v) \leq$ $\pi^{-1}(v d)^{-1 / 2}$.

Let us estimate the boundaries of the support of $F(u)$. From (5) it follows that $\left(2 s_{0}-\right.$ 1) $v \geq \kappa d+O(\varepsilon)$ and as $\varepsilon \rightarrow+0$ we obtain $v(1+2 \sqrt{d / v}) \geq \kappa d+O(\varepsilon)$. Solving this quadratic inequality with respect to $\sqrt{v}$ we find that

$$
v \geq v_{1} \stackrel{\text { def }}{=} \frac{\kappa^{2} d}{4(\sqrt{1+\kappa}+1)^{2}} \quad \text { for } v \in \mathbf{S} .
$$

Further, note that $\mu_{1} \leq \mu_{0} c_{2}$, where $c_{2}$ is an upper bound of the matrices $A^{T} A$ spectra. Also from (5) it follows that

$$
\left(2 s_{0}-1\right) v-\kappa d=p-v \leq c_{2}|s|^{-2}+O(\varepsilon), \quad v \in \mathbf{S} .
$$

We have $s_{0} \geq 1-\sqrt{d / v}+O(\varepsilon)$. Let $v>9 d$. Then $s_{0} \geq 2 / 3+O(\varepsilon)$ and $|s|^{2} \geq 4 / 9+O(\varepsilon)$. Hence $\left(2 s_{0}-1\right) v \leq 9 / 4 c_{2}+\kappa d+O(\varepsilon)$. We estimate $s_{0}$ from below using (6). The inequality follows $v-2 \sqrt{d v} \leq \kappa d+9 / 4 c_{2}$. Solving this inequality with respect to $\sqrt{v}$ as $\varepsilon \rightarrow+0$ we find that

$$
v \leq\left(\sqrt{1+\kappa d+9 / 4 c_{2} / d}+1\right)^{2} d / 4 \text {. }
$$

From this relation a (weaker) upper estimate follows that is presented in the theorem formulation. Thus we have found the boundaries $v_{1}$ and $v_{2}$. Now let us integrate both parts of the inequality $F^{\prime}(v) \leq \pi^{-1}(v d)^{-1 / 2}$. We find that $\sqrt{\pi d} \leq \sqrt{v_{20}}-\sqrt{v_{10}}$, where 
$v_{20} \geq v_{10} \geq 0$ are utmost endpoints of the set $\mathbf{S}$. The lower estimate for the diameter $\mathbf{S}$ follows.

Let us establish the Goelder inequality for $s(z)$. For $\operatorname{Im} z \neq 0$ by (3) the function $h(z)$ is differentiable. Let $v=-\operatorname{Re} z \in \mathbf{S}$. Differentiating left-hand and right-hand sides of (1) we obtain the expression of the derivative $s^{\prime}=s^{\prime}(z)$

$$
s^{\prime} /\left(d s^{2}\right)=-m_{0} s^{\prime}(\kappa d-2 v s)-m_{0} s^{2}, \quad m_{0}=\int(u+r s)^{-2} d F_{0}(u) .
$$

Let us rewrite this equation in the form $s^{\prime}(B-p)=-s^{2}$, where $B=1 /\left(d m_{0} s^{2}\right)$. Now we estimate $\left|s^{\prime}\right|$ from below. By (5) we have $p=1 / \mu_{0} d|s|^{2}+O(\varepsilon)$. If $\kappa>0$ and $z=-v$, where $v \in \mathbf{S}$ then $|s(z)|$ is bounded both from below and from above. For sufficiently small $\varepsilon>0$ we have $p>\delta / 2$ and it follows that $\left|m_{0} s^{2}\right| \leq \mu_{0}|s|^{2}=O(1)$. We find that $\left|s^{\prime}\right| \leq$ const $/|\rho|$, where

$$
\rho=\mu_{0}|s|^{2}-m_{0} s^{2}=\int|u / s+r|^{-2} d F_{0}(u)-\int(u / s+r)^{-2} d F_{0}(u) .
$$

Denote $A=u / s+r$. It is easy to verify that

$$
\operatorname{Re} \rho=2 \int|A|^{-4}(\operatorname{Im} A)^{2} d F_{0}(u) .
$$

The value $\operatorname{Im} A=-|s|^{-2} s_{1}-v s_{1}+O(\varepsilon)$. For sufficiently small $\varepsilon \rightarrow+0$ we have $|\operatorname{Im} A|>$ $v s_{1} / \sqrt{2}$. Applying the Cauchy-Buniakovsky inequality and using (4) we find that if $\varepsilon>0$ is sufficiently small, then

$$
\operatorname{Re} \rho \geq v^{2} s_{1}^{2} \int|A|^{-4} d F_{0}(u) \geq v^{2} s_{1}^{2}|s|^{4} \mu_{0}^{2} \geq(v d)^{2} p^{-2} s_{1}^{2} .
$$

Here $v \geq \delta>0$ and the values $p$ are bounded from above. We conclude that $\left|s^{\prime}\right| \leq$ const $/ s_{1}^{2}$. Consequently, the function $s_{1}^{3}$ has a derivative uniformly bounded in $\mathbf{S}$. As $z \rightarrow-v-i \varepsilon, v<0$ and $\varepsilon \rightarrow+0$ there exists that limit function $s_{1}(-v)=\lim s_{1}(z)$. The difference $s_{1}^{3}(z)-s_{1}^{3}(-v)=O(\varepsilon)$, and $s_{1}(z) \leq s_{1}(-v)+O\left(\varepsilon^{1 / 3}\right)$.

In view of (3) one can conclude that $s_{1}^{3}$ has a uniformly bounded derivative on any compact outside of $\mathbf{S}$. Thus, the function $s_{1}(z)$ satisfies the Goelder inequality with the exponent $1 / 3$ for all $z$

From (3) it follows that for $v>0$ we have $F^{\prime}(v)=\pi^{-1} \operatorname{Im} h(-v)$. One can see that $F^{\prime}(v)$ also satisfies the Lipschitz inequality with the exponent $1 / 3$. If $\kappa>0$ then $v_{1}>0$. It follows that $F^{\prime}(v)=0$ for positive $v<v_{1}$. If $F(v)$ has a jump at the point $v=0$ then the function $h(z)$ must have a pole at the point $z=0$ while it is not greater $1 /(\kappa d)$. One can conclude that if $\kappa>0$, then $F^{\prime}(v)$ satisfies the Lipschitz inequality for all $v \geq 0$. Set $F^{\prime}(v)=0$ for $v<0$. Then $F^{\prime}(v)$ satisfies the Lipschitz inequality for all real $v$.

Now consider Equation (2) as the Cauchy type integral. The integration contour can be extended to the negative half-axis and closed by an infinitely remote circumference. Applying the well-known theorem on integrals of the Cauchy type we can conclude that the function $h(z)$ defined by $(2)$, satisfies the Goelder inequality with the exponent $1 / 3$ on the whole plain of complex $z$. 


\section{Special cases}

Now we study the characteristic features of the limit spectra of matrices $S=R^{T} R$ for a specific case when $F_{0}(v)=$ ind $\left(v \geq a^{2}\right)$. This is true if for each $n$ the matrix $\Sigma=A^{T} A=$ $a^{2} I$. From (2) we obtain

$$
t h s^{2}+h\left(a^{2}-d\right)+\kappa h d s=1,
$$

where $s=1+h d$. Let us introduce a "signal-to-noise" ratio $T=a^{2} / d$.

1. If $T=0$ the matrices $R^{T} R$ are the Wishart matrices, $(s-1)(\kappa-v s)=1$, and one can find the density

$$
F^{\prime}(v)=(2 \pi v)^{-1} \sqrt{\left(v_{2}-v\right)\left(v-v_{1}\right)}
$$

where $v_{1}=(\sqrt{1+\kappa}-1)^{2}, \quad v_{2}=(\sqrt{1+\kappa}+1)^{2}, \quad v_{1} \leq v \leq v_{2}$. This is the well-known Marchenko and Pastur spectral distribution that is characteristic of limit spectra of sample covariance matrices. For $\kappa>0$ the limit spectrum is separated from zero, and for $\kappa=0$ it is located on the segment $[0,4]$.

2. For $\kappa=0$ and $T>0$ for points of the spectrum $\mathbf{S}$ as $\varepsilon \rightarrow+0$ we find that $2 v s_{0}=|s-1|^{-2} d$, where $s_{0}=\operatorname{Re} s$. From Equation (7) one finds

$$
F^{\prime}(v)=\frac{1}{\pi} \operatorname{Im} h(v)=\frac{1}{\pi}\left(x \frac{3 x-2-2(T-1)(1-x)^{2}}{1+2 x(T-1)}\right)^{1 / 2}
$$

where the parameter $x=\operatorname{Re} s \geq 1 / 2$ is determined from the equation

$$
v=\frac{1+2 x(T-1)}{2 x(1-2 x)^{2}} .
$$

For $T \leq 1$ the left endpoint of the limit spectrum $F(v)$ is $v=0$.

Let $T=1$. Then $F^{\prime}(v)=\pi^{-1} \sqrt{x(3 x-2)}$, where $x$ can be calculated from the equation $2 x(1-2 x)^{2} v=1$. In this case the limit spectrum is located on the segment [0,27/4]. For small $v$ the function $F^{\prime}(v) \approx 2 \pi^{-1} \sqrt{3} v^{-1 / 3}, \quad F^{\prime \prime}(27 / 4)=-\infty$.

For $T=1+\varepsilon$, where $\varepsilon>0$ is small, the lower spectrum boundary is near the point $4 / 27 \varepsilon^{3}$; The spectrum is located within boundaries increasing with the increase of $T$.

\section{References}

[1] T. Guhr, A. Muller-Groeling, and H. Weidenmuller, Random matrix theories in quantum physics: common concepts. Physics Rep., 1998, vol.299, 189-425.

[2] V.L. Girko. Statistical Analysis of Observations of Increasing Dimension, Kluwer Academic Publishers, 1995.

[3] V.I. Serdobolskii., Multivariate Statistical Analysis. A High-Dimensional Approach, Kluwer Academic Publishers, Dordrecht, 2000. 